\title{
DAMIEN ROY
}

\section{Sur une version algébrique de la notion de sous- groupe minimal relatif de $\mathbb{R}^{n}$}

\author{
Bulletin de la S. M. F., tome 118, no 2 (1990), p. 171-191 \\ <http://www.numdam.org/item?id=BSMF_1990_118_2_171_0>
}

(C) Bulletin de la S. M. F., 1990, tous droits réservés.

L'accès aux archives de la revue «Bulletin de la S. M. F. » (http: //smf.emath.fr/Publications/Bulletin/Presentation.html) implique l'accord avec les conditions générales d'utilisation (http://www.numdam.org/ conditions). Toute utilisation commerciale ou impression systématique est constitutive d'une infraction pénale. Toute copie ou impression de ce fichier doit contenir la présente mention de copyright.

\section{NumDam}




\title{
SUR UNE VERSION ALGÉBRIQUE DE LA NOTION DE SOUS-GROUPE MINIMAL RELATIF DE $\mathbf{R}^{\mathbf{n}}$
}

\author{
PAR \\ DAMIEN ROY $(*)$
}

\begin{abstract}
RÉSUMÉ. - Nous introduisons, pour des corps $k \subset K$, la notion algébrique de $k$-sous-espace minimal relatif de $K^{n}$, et celle, duale, de $k$-sous-espace étoilé relatif de $K^{n}$. La première traduit, pour $k=\mathbb{Q}$ et $K=\mathbb{R}$, la notion topologique de sousgroupe minimal relatif de $\mathbf{R}^{n}$ rappelée dans l'introduction. Nous établissons certaines propriétés des $k$-sous-espaces étoilés relatifs de $K^{n}$, et, par dualité, nous en déduisons des propriétés correspondantes pour les $k$-sous-espaces minimaux relatifs de $K^{n}$.

ABSTRACT. - We introduce, for fields $k \subset K$, the algebraic notion of a relative minimal $k$-subspace of $K^{n}$, and the dual notion of a relative star-like $k$-subspace of $K^{n}$. The first one traduces, for $k=\mathbb{Q}$ and $K=\mathbb{R}$, the topological notion of a relative minimal subgroup of $\mathbf{R}^{n}$ recalled in the introduction. We obtain properties of relative star-like $k$-subspaces of $K^{n}$, from which, by duality, we deduce corresponding properties for relative minimal $k$-subspaces of $K^{n}$.
\end{abstract}

\section{Introduction}

Soient $H, G$ des sous-groupes de $\mathbb{R}^{n}$ avec $H \subset G$. Dans [3], §1, on introduit les définitions suivantes. On dit que $G$ est un sous-groupe minimal de $\mathbb{R}^{n}$ relativement $\grave{a} H$ si $G$ est de type fini, dense dans $\mathbb{R}^{n}$, et si aucun sous-groupe de $G$ contenant $H$, de rang inférieur au rang de $G$, n'est dense dans $\mathbb{R}^{n}$. On dit aussi que $G$ est un sous-groupe minimal de $\mathbb{R}^{n}$ s'il est minimal relativement à 0 . On dit que $G$ est un sous-groupe étoilé de $\mathbb{R}^{n}$ relativement $\grave{a} H$ si $G$ est de type fini, dense dans $\mathbb{R}^{n}$, et si $G \cap\langle u\rangle_{\mathbf{R}}$ est dense dans $\langle u\rangle_{\mathbf{R}}$ pour tout $u \in H$, où $\langle u\rangle_{\mathbf{R}}$ désigne le sous-espace de $\mathbb{R}^{n}$ engendré par $u$. Enfin, on dit que $G$ est un sous-groupe étoilé de $\mathbb{R}^{n}$ s'il est étoilé relativement à lui-même.

Soient $k$ un corps, et $K$ une extension de $k$. Dans cet article, on définit les notions analogues de $k$-sous-espace minimal relatif de $K^{n}$, de

(*) Texte reçu le 28 juin 1989 , révisé le 30 août 1990 .

D. RoY, Institut Henri-Poincaré, Problèmes diophantiens, 11, rue Pierre-et-MarieCurie, 75231 Paris Cedex 05, France. 
$k$-sous-espace étoilé relatif de $K^{n}$, de $k$-sous-espace minimal de $K^{n}$, et de $k$-sous-espace étoilé de $K^{n}$. Le cas important est celui où $k=\mathbb{Q}$ et $K=\mathbb{R}$. En effet, désignons par $\langle H\rangle_{\mathbb{Q}}$ et $\langle G\rangle_{\mathbb{Q}}$ les $\mathbb{Q}$-sous-espaces de $\mathbb{R}^{n}$ engendrés respectivement par $H$ et $G$. Les nouvelles définitions sont données de telle sorte que $G$ soit un sous-groupe minimal (resp. étoilé) de $\mathbb{R}^{n}$ relativement à $H$ si et seulement si $\langle G\rangle_{\mathbb{Q}}$ est un $\mathbb{Q}$-sous-espace minimal (resp. étoilé) de $\mathbb{R}^{n}$ relativement à $\langle H\rangle_{\mathbb{Q}}$. Il s'ensuit que $G$ est un sous-groupe minimal (resp. étoilé) de $\mathbb{R}^{n}$ si et seulement si $\langle G\rangle_{\mathbb{Q}}$ est un $\mathbb{Q}$-sous-espace minimal (resp. étoilé) de $\mathbb{R}^{n}$.

Dans le nouveau contexte, des conditions algébriques sont substituées aux conditions topologiques. Pour ce faire, on transpose la notion de sousgroupe dense de $\mathbb{R}^{n}$, grâce à la caractérisation qu'en donne [1], $\S 1, \mathrm{n}^{\circ} 3$. On obtient ainsi la notion de $k$-sous-espace de type (D) de $K^{n}$, sur laquelle se fondent les autres notions.

Le but de ce travail est d'établir certaines propriétés des $k$-sous-espaces minimaux relatifs et étoilés relatifs de $K^{n}$. Ces propriétés se traduisent immédiatement en des propriétés analogues des sous-groupes minimaux relatifs et étoilés relatifs de $\mathbb{R}^{n}$. On démontre ainsi les propositions énoncées sans démonstration au $\S 1$ de [3]. Par ailleurs, cet article ne s'appuie pas sur [3]. Cependant, on trouvera dans [3] des questions qui motivent l'étude des sous-groupes minimaux relatifs de $\mathbb{R}^{n}$.

Le $\S 1$ de cet article regroupe les définitions. Au $\S 2$, on montre qu'il y a dualité entre les notions de $k$-sous-espace minimal relatif et de $k$ sous-espace étoilé relatif. Ainsi l'étude de la première se ramène à celle de la seconde. Pour la suite, on est amené à supposer que le corps $k$ est parfait, de cardinalité infinie. Au $\S 3$, on montre que la dimension d'un $k$-sous-espace étoilé de $K^{n}$ est $\geq 2 n$. On donne, pour $n \geq 2$, une caractérisation des $k$-sous-espaces étoilés de $K^{n}$, et des $k$-sous-espaces de $K^{n}$ étoilés relativement à un $k$-sous-espace de codimension 1 . La première fait voir qu'à chaque $k$-sous-espace étoilé de $K^{n}$ répond une extension algébrique de $k$, contenue dans $K$, distincte de $k$. Au $\S 4$, on en déduit que la dimension d'un $k$-sous-espace minimal de $K^{n}$ est $\leq 2 n$, et qu'elle est égale à $n+1$ si $k$ est algébriquement clos dans $K$. On obtient aussi une description des $k$-sous-espaces minimaux de $K^{n}$ de dimension $n+1,2 n-1$ et $2 n$. Ainsi, on montre que, pour $n \geq 2$, un $k$-sous-espace $E$ de $K^{n}$ de dimension $2 n$ est minimal si et seulement s'il existe une extension quadratique $F$ de $k$ contenue dans $K$, et une base de $K^{n}$ sur $K$, tels que $E$ soit le $F$-sous-espace de $K^{n}$ engendré par cette base. Enfin, on donne, pour $n \geq 2$, une caractérisation des $k$-sous-espaces de $K^{n}$ de dimension $\geq n+2$, minimaux relativement à un $k$-sous-espace de dimension 1 . On conclut par un critère de minimalité.

$$
\text { TOME } 118-1990-\mathrm{N}^{\circ} 2
$$


La poursuite de cette recherche, commencée au doctorat avec le support d'une bourse doctorale du FCAR (Québec), a été rendue possible grâce au soutien d'une bourse en sciences de l'OTAN du CRSNG (Canada). Elle a été accomplie au cours d'un stage au sein de l'équipe "Problèmes diophantiens" de l'université Paris VI, que je remercie de son accueil chaleureux.

\section{Notations}

Soit $F$ un corps quelconque. Pour chaque entier $n \geq 0$, on munit le $F$ espace vectoriel $F^{n}$ de sa forme bilinéaire standard, notée $(,)_{n}$. Par suite, la transposée d'une application $F$-linéaire $f: F^{m} \rightarrow F^{n}$ est définie comme l'application $F$-linéaire ${ }^{t} f: F^{n} \rightarrow F^{m}$ vérifiant $\left(x,{ }^{t} f(y)\right)_{m}=(f(x), y)_{n}$ pour tout $x \in F^{m}$ et $y \in F^{n}$. Si $U$ est un $F$-sous-espace de $F^{n}$, on note $U^{\perp}$ le $F$-sous-espace de $F^{n}$ orthogonal à $U$. On dit que deux sousespaces de $F^{n}$ sont supplémentaires orthogonaux si l'un est l'orthogonal de l'autre par rapport à $(,)_{n}$. Si $V$ est un $F$-espace vectoriel, et $S$ un sous-ensemble de $V$, on note $\langle S\rangle_{F}$ le $F$-sous-espace de $V$ engendré par $S$.

Dans la suite, on fixe le choix d'un corps $k$ et d'une extension $K$ de $k$. Si $f: k^{m} \rightarrow k^{n}$ est une application $k$-linéaire, on note $f_{K}$ l'unique application $K$-linéaire de $K^{m}$ dans $K^{n}$ qui coïncide avec $f$ sur $k^{m}$. Alors on a ${ }^{t}\left(f_{K}\right)=\left({ }^{t} f\right)_{K}$. Si $m=n$ et si $f$ est un isomorphisme, alors ${ }^{t} f$ et $f_{K}$ en sont aussi. On a $\left({ }^{t} f\right)^{-1}={ }^{t}\left(f^{-1}\right)$ et $\left(f_{K}\right)^{-1}=\left(f^{-1}\right)_{K}$. Ainsi, pour un automorphisme $f$ de $k^{n}$, les trois opérations : passage à l'inverse, passage à la transposée, et passage de $f$ à $f_{K}$, commutent entre elles, et l'expression ${ }^{t} f_{K}^{-1}$ n'est pas ambiguë. Les lettres $\mathbb{Q}$ et $\mathbb{R}$ désignent les corps usuels.

\section{Définitions}

Soient $n, n_{1}, n_{2}$ des entiers positifs. On introduit d'abord trois définitions :

Définition. - Soit $E$ un $k$-sous-espace de $K^{n}$. On définit le $k$-sousespace de $K^{n}$ associé à $E$ par

$$
E_{\text {ass } / k}=\left\{x \in K^{n} ;(x, E)_{n} \subset k\right\}
$$

On dit que $E$ est un $k$-sous-espace de type (D) de $K^{n}$ si $E_{\text {ass } / k}=0$.

Définition. - On dit qu'un $k$-sous-espace $E$ de $K^{n}$ est minimal relativement $\grave{a}$ un $k$-sous-espace $B \subset E$, s'il est de dimension finie, de type (D), et ne contient aucun $k$-sous-espace de type (D) de $K^{n}$, 
contenant $B$, de dimension inférieure. On dit qu'il est minimal, s'il est minimal relativement à 0 .

Définition. - On dit qu'un $k$-sous-espace $E$ de $K^{n}$ est étoilé relativement à un $k$-sous-espace $B \subset E$, s'il est de dimension finie, de type (D), et si la dimension de $E \cap\langle u\rangle_{K}$ sur $k$ est $\geq 2$ pour tout $u \in B$ non nul. On dit qu'il est étoilé s'il est étoilé relativement à lui-même.

Soient $H, G$ des sous-groupes de type fini de $\mathbb{R}^{n}$, avec $H \subset G$. On pose $B=\langle H\rangle_{\mathbb{Q}}$ et $E=\langle G\rangle_{\mathbb{Q}}$. D'après [1], $\S 1, \mathrm{n}^{\circ} 3$, le groupe $G$ est dense dans $\mathbb{R}^{n}$ si et seulement si son sous-groupe associé

$$
G_{\text {ass }}=\left\{x \in \mathbb{R}^{n} ;(x, G)_{n} \subset \mathbb{Z}\right\}
$$

est réduit à $\{0\}$. On a $E_{\text {ass } / \mathbb{Q}}=\left\langle G_{\text {ass }}\right\rangle_{\mathbb{Q}}$. On en déduit que $G$ est dense dans $\mathbb{R}^{n}$ si et seulement si $E$ est un $\mathbb{Q}$-sous-espace de type (D) de $\mathbb{R}^{n}$. En appliquant ce résultat à l'ensemble des sous-groupes de $G$ contenant $H$, on obtient que $G$ est un sous-groupe minimal de $\mathbb{R}^{n}$ relativement à $H$ si et seulement si $E$ est un $\mathbb{Q}$-sous-espace minimal de $\mathbb{R}^{n}$ relativement à $B$. D'autre part, pour $u \in \mathbb{R}^{n}$, avec $u \neq 0$, le groupe $G \cap\langle u\rangle_{\mathbb{R}}$ est dense dans $\langle u\rangle_{\mathbb{R}}$ si et seulement si $E \cap\langle u\rangle_{\mathbb{R}}$ est de dimension $\geq 2$ sur $\mathbb{Q}$. On en déduit que $G$ est un sous-groupe étoilé de $\mathbb{R}^{n}$ relativement à $H$ si et seulement si $E$ est un $\mathbb{Q}$-sous-espace étoilé de $\mathbb{R}^{n}$ relativement à $B$. En particulier, $G$ est un sous-groupe minimal (resp. étoilé) de $\mathbb{R}^{n}$ si et seulement si $E$ est un $\mathbb{Q}$-sous-espace minimal (resp. étoilé) de $\mathbb{R}^{n}$. Enfin, $H$ est un sous-groupe discret de $\mathbb{R}^{n}$ si et seulement si $B$ est engendré par des éléments de $\mathbb{R}^{n}$ linéairement indépendants $\operatorname{sur} \mathbb{R}\left([1], \S 1, \mathrm{n}^{\circ} 1\right)$.

Ces remarques permettent de transcrire, en termes de sous-groupes de $\mathbb{R}^{n}$, les résultats qui seront énoncés par la suite en termes de $k$-sousespaces de $K^{n}$. De telles transcriptions sont données dans [3], $\S 1$. Les informations qu'elles livrent sur les sous-groupes minimaux relatifs de $\mathbb{R}^{n}$ sont employées dans [3] à l'étude des sous-groupes minimaux des groupes de Lie commutatifs réels, la composante neutre d'un tel groupe de Lie étant isomorphe, pour un entier $n \geq 0$, au quotient de $\mathbb{R}^{n}$ par un sousgroupe discret.

On introduit maintenant une relation d'équivalence sur l'ensemble des $k$-sous-espaces de dimension finie de $K^{n}$, et sur l'ensemble des couples $(B, E)$ de $k$-sous-espaces de dimension finie de $K^{n}$, avec $B \subset E$. On introduit aussi une notion de dualité.

Définition. - Soient $E_{1}, E_{2}$ des $k$-sous-espaces de $K^{n}$ de dimension finie. On dit qu'ils sont équivalents s'il existe un automorphisme de $K^{n}$ qui applique $E_{1}$ sur $E_{2}$.

$$
\text { TOME } 118-1990-\mathrm{N}^{\circ} 2
$$


Soient $B_{1}$ un sous-espace de $E_{1}$, et $B_{2}$ un sous-espace de $E_{2}$. On dit que les couples $\left(B_{1}, E_{1}\right)$ et $\left(B_{2}, E_{2}\right)$ sont équivalents s'il existe un automorphisme de $K^{n}$ qui applique $B_{1}$ sur $B_{2}$ et $E_{1} \operatorname{sur} E_{2}$.

Définition. - Soient $E_{1}$ un $k$-sous-espace de $K^{n_{1}}$ de dimension finie, et $E_{2}$ un $k$-sous-espace de $K^{n_{2}}$ de dimension finie. On dit qu'ils sont duaux s'ils ont même dimension $m=n_{1}+n_{2}$, et s'il existe des applications linéaires surjectives $f_{1}: K^{m} \rightarrow K^{n_{1}}, f_{2}: K^{m} \rightarrow K^{n_{2}}$, de noyaux supplémentaires orthogonaux, telles que $f_{1}\left(k^{m}\right)=E_{1}$ et $f_{2}\left(k^{m}\right)=E_{2}$.

Soient $B_{1}$ un sous-espace de $E_{1}$, et $B_{2}$ un sous-espace de $E_{2}$. On dit que les couples $\left(B_{1}, E_{1}\right)$ et $\left(B_{2}, E_{2}\right)$ sont duaux si $E_{1}$ et $E_{2}$ ont même dimension $m=n_{1}+n_{2}$, et s'il existe des applications linéaires $f_{1}, f_{2}$ comme ci-dessus, et des sous-espaces $U_{1}, U_{2}$ de $k^{m}$, supplémentaires orthogonaux dans $k^{m}$, tels qu'on ait en plus $f_{1}\left(U_{1}\right)=B_{1}$ et $f_{2}\left(U_{2}\right)=B_{2}$.

\section{2. Équivalence et dualité}

On va montrer que les caractères "minimal" et "étoilé" sont conservés sous l'équivalence, et qu'ils s'échangent sous la dualité. Ce seront les Propositions 2.1 et 2.5. On verra, par la Proposition 2.7, que, lorsqu'il existe, le dual est défini à équivalence près. De ces trois propositions découlent respectivement les propositions 1.1, 1.2 et 1.3 de [3]. Enfin, on verra, dans la Proposition 2.8, une forme concrète de ces résultats pour le cas absolu.

Proposition 2.1. - Soient $n$ un entier positif, et $E_{1}, E_{2}$ des $k$-sousespaces de dimension finie de $K^{n}$. Supposons qu'ils soient équivalents. Alors $E_{1}$ et $E_{2}$ ont même dimension. De plus, si $E_{1}$ est un $k$-sous-espace de type (D), minimal, ou étoilé de $K^{n}$, alors $E_{2}$ l'est aussi.

Soient $B_{1}$ un sous-espace de $E_{1}$, et $B_{2}$ un sous-espace de $E_{2}$. Supposons que les couples $\left(B_{1}, E_{1}\right)$ et $\left(B_{2}, E_{2}\right)$ soient équivalents. Alors $E_{1}$ et $E_{2}$ ont même dimension. Si $B_{1}$ est engendré par des éléments de $K^{n}$ linéairement indépendants sur $K$, ou s'il contient une base de $K^{n}$ sur $K$, la même chose vaut pour $B_{2}$. Si $E_{1}$ est de type (D), $E_{2}$ l'est aussi. Enfin, si $E_{1}$ est un $k$-sous-espace minimal ou étoilé de $K^{n}$ relativement à $B_{1}$, alors $E_{2}$ est dans la même relation par rapport à $B_{2}$.

Démonstration. - Supposons que $\left(B_{1}, E_{1}\right)$ et $\left(B_{2}, E_{2}\right)$ soient équivalents. Il existe un automorphisme $\Psi$ de $K^{n}$ tel que $\Psi\left(B_{2}\right)=B_{1}$ et $\Psi\left(E_{2}\right)=E_{1}$. Par restriction $\Psi$ détermine un $k$-isomorphisme de $E_{2}$ sur $E_{1}$. Alors la correspondance $E \mapsto \Psi(E)$ fournit une bijection entre les $k$-sousespaces de $E_{2}$ contenant $B_{2}$ et ceux de $E_{1}$ contenant $B_{1}$. Elle préserve 
la dimension sur $k$, et vérifie $E_{\text {ass } / k}={ }^{t} \Psi\left(\left((\Psi(E))_{\text {ass } / k}\right)\right.$. Donc un sousespace $E$ de $E_{2}$ est de type (D) si $\Psi(E)$ l'est. On en déduit que $E_{2}$ est de type (D) si $E_{1}$ l'est, et que $E_{2}$ est minimal relativement à $B_{2}$ si $E_{1}$ est minimal relativement à $B_{1}$. On a aussi $\Psi\left(E_{2} \cap\langle u\rangle_{K}\right)=E_{1} \cap\langle\Psi(u)\rangle_{K}$ pour tout $u \in B_{2}$. On en déduit que $E_{2}$ est étoilé relativement à $B_{2}$ si $E_{1}$ est étoilé relativement à $B_{1}$. Cela démontre la seconde partie de la proposition, à l'exception de la seconde assertion, qui est immédiate.

Enfin, supposons que $E_{1}$ et $E_{2}$ soient équivalents. Alors les couples $\left(0, E_{1}\right)$ et $\left(0, E_{2}\right)$ sont équivalents. En vertu de la seconde partie de la proposition, cela implique que $E_{1}, E_{2}$ ont même dimension sur $k$, et que, si $E_{1}$ est de type (D) ou minimal, $E_{2}$ l'est aussi. Les couples $\left(E_{1}, E_{1}\right)$ et $\left(E_{2}, E_{2}\right)$ aussi sont équivalents. Alors $E_{2}$ est étoilé si $E_{1}$ l'est.

Lemme 2.2. - Soient $n_{1}, n_{2}$ des entiers positifs, $m$ leur somme, et $f_{1}: K^{m} \rightarrow K^{n_{1}}, f_{2}: K^{m} \rightarrow K^{n_{2}}$ des applications linéaires surjectives de noyaux supplémentaires orthogonaux. On pose $E_{1}=f_{1}\left(k^{m}\right)$ et $E_{2}=$ $f_{2}\left(k^{m}\right)$. Alors $E_{1}$ est un $k$-sous-espace de type (D) de $K^{n_{1}}$ si et seulement si $E_{2}$ est un $k$-sous-espace de dimension $m$ de $K^{n_{2}}$.

Démonstration. - Pour tout $x \in K^{n_{1}}$, on a $\left(x, E_{1}\right)_{n_{1}}=\left({ }^{t} f_{1}(x), k^{m}\right)_{m}$. Donc la condition $x \in\left(E_{1}\right)_{\text {ass } / k}$ équivaut à ${ }^{t} f_{1}(x) \in k^{m}$. On en déduit ${ }^{t} f_{1}\left(\left(E_{1}\right)_{\text {ass } / k}\right)={ }^{t} f_{1}\left(K^{n_{1}}\right) \cap k^{m}$. Or, d'une part, ${ }^{t} f_{1}$ est injective, puisque $f_{1}$ est surjective. D'autre part, on a ${ }^{t} f_{1}\left(K^{n_{1}}\right)=\operatorname{ker} f_{2}$. Donc $E_{1}$ est un $k$ sous-espace de type (D) de $K^{n_{1}}$ si et seulement si $f_{2}$ est injective sur $k^{m}$, c'est-à-dire si et seulement si $\operatorname{dim}_{k} E_{2}=m$.

Lemme 2.3. - Avec les notations du Lemme 2.2, supposons que $E_{2}$ soit de dimension $m$ sur $k$. Soient $x$ un élément non nul de $k^{m}$, et $x^{\perp}$ le sous-espace de $k^{m}$ orthogonal à $x$. Alors le sous-espace $f_{1}\left(x^{\perp}\right)$ de $E_{1} n^{\prime}$ 'est pas de type (D) dans $K^{n_{1}}$ si et seulement si la dimension de $E_{2} \cap\left\langle f_{2}(x)\right\rangle_{K}$ sur $k$ est $\geq 2$.

Démonstration. - Puisque $\operatorname{dim}_{k} E_{2}=m$, l'application $f_{2}$ est injective sur $k^{m}$, et on a $f_{2}(x) \neq 0$. Soit $y \in k^{m}$ tel que $(x, y)_{m}=1$. On pose $M=\left\{t \in K ; t f_{2}(x) \in E_{2}\right\}$, et on considère l'application $k$-linéaire $\theta:\left(f_{1}\left(x^{\perp}\right)\right)_{\text {ass } / k} \rightarrow K / k$ donnée $\operatorname{par} \theta(z)=\left(z, f_{1}(y)\right)_{n_{1}}+k$. Pour conclure, il suffit de montrer qu'elle est injective d'image égale à $M / k$.

On a $k^{m}=x^{\perp}+\langle y\rangle_{k}$, donc $E_{1}=f_{1}\left(x^{\perp}\right)+\left\langle f_{1}(y)\right\rangle_{k}$. Alors le noyau de $\theta$ est $\left(E_{1}\right)_{\text {ass } / k}$. Comme $E_{1}$ est de type (D) dans $K^{n_{1}}$ en vertu du LEMme 2.2, cela signifie que $\theta$ est injective.

Pour tout $z \in K^{n_{1}}$ et tout $t \in K$, les conditions $z \in\left(f_{1}\left(x^{\perp}\right)\right)_{\text {ass } / k}$ et $\theta(z)=t+k$ équivalent à $\left({ }^{t} f_{1}(z), x^{\perp}\right)_{m} \subset k$ et $\left({ }^{t} f_{1}(z), y\right)_{m}-t \in k$,

TOME $118-1990-\mathrm{N}^{\circ} 2$ 
ou encore à $\left({ }^{t} f_{1}(z)-t x, k^{m}\right)_{m} \subset k$, ou encore à ${ }^{t} f_{1}(z)-t x \in k^{m}$. Donc un élément $t+k$ de $K / k$ appartient à l'image de $\theta$ si et seulement si $t x \in k^{m}+{ }^{t} f_{1}\left(K^{n_{1}}\right)$. Comme ${ }^{t} f_{1}\left(K^{n_{1}}\right)=$ ker $f_{2}$, cette dernière condition équivaut à $t f_{2}(x) \in E_{2}$. Donc l'image de $\theta$ est $M / k$.

LEMmE 2.4. - En conservant les notations du LEMME 2.2, supposons que $E_{1}$ soit de dimension $m$ sur $k$. Soient $U_{1}$ et $U_{2}$ des sous-espaces supplémentaires orthogonaux de $k^{m}$. On pose $B_{1}=f_{1}\left(U_{1}\right)$ et $B_{2}=$ $f_{2}\left(U_{2}\right)$. Alors $B_{1}$ est engendré par des éléments de $K^{n_{1}}$ linéairement indépendants sur $K$ si et seulement si $\left\langle B_{2}\right\rangle_{K}=K^{n_{2}}$.

Démonstration. - Puisque $\operatorname{dim}_{k} E_{2}=m$, l'application $f_{1}$ est injective sur $k^{m}$, et on a $\operatorname{dim}_{k} B_{1}=\operatorname{dim}_{k} U_{1}$. On a aussi $\left\langle B_{1}\right\rangle_{K}=f_{1}\left(\left\langle U_{1}\right\rangle_{K}\right)$ et $\operatorname{dim}_{K}\left\langle U_{1}\right\rangle_{K}=\operatorname{dim}_{k} U_{1}$. Donc la condition que $B_{1}$ soit engendré par des éléments de $K^{n_{1}}$ linéairement indépendants sur $K$, qui se traduit par $\operatorname{dim}_{k} B_{1}=\operatorname{dim}_{K}\left\langle B_{1}\right\rangle_{K}$, équivaut à l'injectivité de $f_{1}$ sur $\left\langle U_{1}\right\rangle_{K}$. Puisque $\left(\left(\operatorname{ker} f_{1}\right) \cap\left\langle U_{1}\right\rangle_{K}\right)^{\perp}=\left(\operatorname{ker} f_{2}\right)+\left\langle U_{2}\right\rangle_{\dot{K}}$, cette condition équivaut encore à $\left(\operatorname{ker} f_{2}\right)+\left\langle U_{2}\right\rangle_{K}=K^{m}$. Comme $f_{2}$ est surjective, et que $f_{2}\left(\left\langle U_{2}\right\rangle_{K}\right)=\left\langle B_{2}\right\rangle_{K}$, cela revient à demander $\left\langle B_{2}\right\rangle_{K}=K^{n_{2}}$.

On en déduit :

Proposition 2.5. - Soient $n_{1}, n_{2}$ des entiers positifs, et $m$ leur somme. Soient $E_{1}$ un $k$-sous-espace de dimension finie de $K^{n_{1}}$, et $E_{2}$ un $k$-sous-espace de dimension finie de $K^{n_{2}}$. Supposons qu'ils soient duaux. Alors $E_{1}$ et $E_{2}$ sont de type (D) et de dimension $m$. De plus $E_{1}$ est un $k$-sous-espace minimal de $K^{n_{1}}$ si et seulement si $E_{2}$ est un $k$-sous-espace étoilé de $K^{n_{2}}$.

Soient $B_{1}$ un sous-espace de $E_{1}$, et $B_{2}$ un sous-espace de $E_{2}$. Supposons maintenant que les couples $\left(B_{1}, E_{1}\right)$ et $\left(B_{2}, E_{2}\right)$ soient duaux. Alors $E_{1}$ et $E_{2}$ sont de type (D) et de dimension $m$. On a $\operatorname{dim}_{k} B_{1}+\operatorname{dim}_{k} B_{2}=m$. De plus $B_{1}$ est engendré par des éléments de $K^{n_{1}}$ linéairement indépendants sur $K$ si et seulement si $\left\langle B_{2}\right\rangle_{K}=K^{n_{2}}$. Enfin, $E_{1}$ est un $k$-sousespace minimal de $K^{n_{1}}$ relativement à $B_{1}$ si et seulement si $E_{2}$ est un $k$-sous-espace étoilé de $K^{n_{2}}$ relativement à $B_{2}$.

Démonstration. - La première partie de la proposition découle de la seconde. En effet, si $E_{1}$ et $E_{2}$ sont duaux, alors les couples $\left(0, E_{1}\right)$ et $\left(E_{2}, E_{2}\right)$ sont duaux, et la conclusion suit.

Supposons donc que $\left(B_{1}, E_{1}\right)$ et $\left(B_{2}, E_{2}\right)$ soient duaux. Alors $E_{1}, E_{2}$ ont pour dimension $m$, et il existe des applications linéaires surjectives $f_{1}: K^{m} \rightarrow K^{n_{1}}, f_{2}: K^{m} \rightarrow K^{n_{2}}$ de noyaux supplémentaires orthogonaux, et des sous-espaces $U_{1}, U_{2}$ de $k^{m}$, supplémentaires orthogonaux dans $k^{m}$, 
tels que $f_{1}\left(U_{1}\right)=B_{1}, f_{1}\left(k^{m}\right)=E_{1}, f_{2}\left(U_{2}\right)=B_{2}$ et $f_{2}\left(k^{m}\right)=E_{2}$. Puisque $f_{1}$ et $f_{2}$ sont injectives sur $k^{m}$, on a $\operatorname{dim}_{k} B_{1}+\operatorname{dim}_{k} B_{2}=\operatorname{dim}_{k} U_{1}+$ $\operatorname{dim}_{k} U_{2}=m$. En vertu du Lemme 2.2, $E_{1}$ et $E_{2}$ sont de type (D). Donc pour que $E_{1}$ soit un $k$-sous-espace minimal de $K^{n_{1}}$ relativement à $B_{1}$, il faut et il suffit que, pour tout $x \in U_{2}$ non nul, $f_{1}\left(x^{\perp}\right)$ ne soit pas un $k$-sous-espace de type (D) de $K^{n_{1}}$, où $x^{\perp}$ désigne le sous-espace de $k^{m}$ orthogonal à $x$. De même, pour que $E_{2}$ soit un $k$-sous-espace étoilé de $K^{n_{2}}$ relativement à $B_{2}$, il faut et il suffit que, pour tout $x \in U_{2}$ non nul, la dimension de $E_{2} \cap\left\langle f_{2}(x)\right\rangle_{K}$ sur $k$ soit $\geq 2$. Suivant le Lemme 2.3 , ces deux conditions sont équivalentes. Enfin le Lemme 2.4 montre que $B_{1}$ est engendré par des éléments de $K^{n_{1}}$ linéairement indépendants sur $K$ si et seulement si $\left\langle B_{2}\right\rangle_{K}=K^{n_{2}}$.

Lemme 2.6. - Soient $n_{1}, n_{2}$ des entiers positifs, $B_{1} \subset E_{1}, B_{1}^{\prime} \subset E_{1}^{\prime}$ des $k$-sous-espaces de dimension finie de $K^{n_{1}}$, et $B_{2} \subset E_{2}, B_{2}^{\prime} \subset E_{2}^{\prime}$ des $k$-sous-espaces de dimension finie de $K^{n_{2}}$.

(i) Supposons que $\left(B_{1}, E_{1}\right)$ et $\left(B_{1}^{\prime}, E_{1}^{\prime}\right)$ soient équivalents, de même que $\left(B_{2}, E_{2}\right)$ et $\left(B_{2}^{\prime}, E_{2}^{\prime}\right)$. Alors $\left(B_{1}, E_{1}\right)$ et $\left(B_{2}, E_{2}\right)$ sont duaux si et seulement si $\left(B_{1}^{\prime}, E_{1}^{\prime}\right)$ et $\left(B_{2}^{\prime}, E_{2}^{\prime}\right)$ sont duaux.

(ii) Supposons que $\left(B_{1}, E_{1}\right)$ et $\left(B_{2}, E_{2}\right)$ soient duaux, de même que $\left(B_{1}^{\prime}, E_{1}^{\prime}\right)$ et $\left(B_{2}^{\prime}, E_{2}^{\prime}\right)$. Alors $\left(B_{1}, E_{1}\right)$ et $\left(B_{1}^{\prime}, E_{1}^{\prime}\right)$ sont équivalents si et seulement si $\left(B_{2}, E_{2}\right)$ et $\left(B_{2}^{\prime}, E_{2}^{\prime}\right)$ sont équivalents.

\section{Démonstration.}

(i) Par hypothèse, il existe des automorphismes $\Psi_{1}$ de $K^{n_{1}}$ et $\Psi_{2}$ de $K^{n_{2}}$ tels que $\Psi_{i}\left(B_{i}\right)=B_{i}^{\prime}$ et $\Psi_{i}\left(E_{i}\right)=E_{i}^{\prime}$ pour $i=1$, 2. Supposons que $\left(B_{1}, E_{1}\right)$ et $\left(B_{2}, E_{2}\right)$ soient duaux. Alors $E_{1}$ et $E_{2}$ ont même dimension $m=n_{1}+n_{2}$, et il existe des applications linéaires surjectives $f_{1}: K^{m} \rightarrow K^{n_{1}}, f_{2}: K^{m} \rightarrow K^{n_{2}}$, de noyaux supplémentaires orthogonaux, et des sous-espaces $U_{1}, U_{2}$ de $k^{m}$, supplémentaires orthogonaux dans $k^{m}$, tels que $f_{i}\left(U_{i}\right)=B_{i}$ et $f_{i}\left(k^{m}\right)=E_{i}$ pour $i=1,2$. On pose $f_{1}^{\prime}=\Psi_{1} \circ f_{1}$ et $f_{2}^{\prime}=\Psi_{2} \circ f_{2}$. Alors $f_{1}^{\prime}$ et $f_{2}^{\prime}$ sont surjectives, et leurs noyaux sont supplémentaires orthogonaux. On a $f_{i}^{\prime}\left(U_{i}\right)=B_{i}^{\prime}$ et $f_{i}^{\prime}\left(k^{m}\right)=E_{i}^{\prime}$ pour $i=1,2$. De plus $E_{1}^{\prime}$ et $E_{2}^{\prime}$ sont aussi de dimension $m$. Donc les couples $\left(B_{1}^{\prime}, E_{1}^{\prime}\right)$ et $\left(B_{2}^{\prime}, E_{2}^{\prime}\right)$ sont duaux. La réciproque se démontre de la même façon.

(ii) Par hypothèse, $E_{1}, E_{2}, E_{1}^{\prime}, E_{2}^{\prime}$ ont même dimension $m=n_{1}+n_{2}$, et il existe des paires d'applications linéaires surjectives $f_{1}: K^{m} \rightarrow K^{n_{1}}$, $f_{2}: K^{m} \rightarrow K^{n_{2}}$ et $f_{1}^{\prime}: K^{m} \rightarrow K^{n_{1}}, f_{2}^{\prime}: K^{m} \rightarrow K^{n_{2}}$, de noyaux supplémentaires orthogonaux, et des paires de sous-espaces $U_{1}, U_{2}$ et $U_{1}^{\prime}, U_{2}^{\prime}$ de $k^{m}$, supplémentaires orthogonaux dans $k^{m}$, tels que $f_{i}\left(U_{i}\right)=B_{i}, f_{i}\left(k^{m}\right)=E_{i}$, $f_{i}^{\prime}\left(U_{i}^{\prime}\right)=B_{i}^{\prime}$ et $f_{i}^{\prime}\left(k^{m}\right)=E_{i}^{\prime}$, pour $i=1,2$. Supposons que $\left(B_{1}, E_{1}\right)$ 
et $\left(B_{1}^{\prime}, E_{1}^{\prime}\right)$ soient équivalents. Alors il existe un automorphisme $\Psi_{1}$ de $K^{n_{1}}$ tel que $\Psi_{1}\left(B_{1}\right)=B_{1}^{\prime}$ et $\Psi_{1}\left(E_{1}\right)=E_{1}^{\prime}$. Puisque $f_{1}$ et $f_{1}^{\prime}$ déterminent par restriction des isomorphismes de $k^{m}$ sur $E_{1}$ et $E_{1}^{\prime}$ respectivement, il existe un automorphisme $\psi$ de $k^{m}$ tel que $f_{1}^{\prime} \circ \psi$ coïncide avec $\Psi_{1} \circ f_{1}$ sur $k^{m}$. Par linéarité, cela implique $f_{1}^{\prime} \circ \psi_{K}=\Psi_{1} \circ f_{1}$. De cette égalité, on tire $\operatorname{ker} f_{1}^{\prime}=\psi_{K}\left(\operatorname{ker} f_{1}\right)$, d'où

$$
\operatorname{ker} f_{2}^{\prime}=\left(\psi_{K}\left(\operatorname{ker} f_{1}\right)\right)^{\perp}={ }^{t} \psi_{K}^{-1}\left(\left(\operatorname{ker} f_{1}\right)^{\perp}\right)={ }^{t} \psi_{K}^{-1}\left(\operatorname{ker} f_{2}\right) .
$$

Cela implique l'existence d'un automorphisme $\Psi_{2}$ de $K^{n_{2}}$ tel que $\Psi_{2} \circ f_{2}^{\prime}=$ $f_{2}{ }^{t} \psi_{K}$. En comparant l'image de $k^{m}$ sous chacun des deux membres de cette égalité, on obtient $\Psi_{2}\left(E_{2}^{\prime}\right)=E_{2}$, $\operatorname{car}^{t} \psi$ est un automorphisme de $k^{m}$. En comparant les images de $U_{2}^{\prime}$, on obtient de même $\Psi_{2}\left(B_{2}^{\prime}\right)=B_{2}$. En effet, comme le choix de $\psi$ implique $\psi\left(U_{1}\right)=U_{1}^{\prime}$, on a, dans $k^{m}$,

$$
U_{2}^{\prime}=\left(\psi\left(U_{1}\right)\right)^{\perp}={ }^{t} \psi^{-1}\left(U_{1}^{\perp}\right)={ }^{t} \psi^{-1}\left(U_{2}\right) .
$$

Donc les couples $\left(B_{2}, E_{2}\right)$ et $\left(B_{2}^{\prime}, E_{2}^{\prime}\right)$ sont équivalents. La réciproque se démontre de la même façon.

Proposition 2.7. - Soient $n$ un entier positif, et $E$ un $k$-sous-espace de $K^{n}$ de dimension finie $m$. Pour que $E$ admette un dual, il faut et il suffit qu'il soit de type (D). Alors $m$ est $>n$, et les duaux de $E$ sont des $k$-sous-espaces de type (D) de $K^{m-n}$, de dimension $m$. Ils constituent une classe d'équivalence dont chaque élément est dual à chaque élément de la classe d'équivalence de $E$.

Soit $B$ un sous-espace de $E$ de dimension $q$. Pour que le couple $(B, E)$ admette un dual, il faut et il suffit que $E$ soit de type (D). Alors $m$ est $>n$, et les duaux de $(B, E)$ sont des couples $\left(B^{\prime}, E^{\prime}\right)$ de $k$-sous-espaces de $K^{m-n}$, avec $B^{\prime} \subset E^{\prime}, B^{\prime}$ de dimension $m-q$, et $E^{\prime}$ de type (D), de dimension $m$. Ils constituent une classe d'équivalence dont chaque élément est dual à chaque élément de la classe d'équivalence de $(B, E)$.

Démonstration. - La première partie de la proposition découle de la seconde, en fonction des observations suivantes. Les couples duaux à $(0, E)$ sont les couples $\left(E^{\prime}, E^{\prime}\right)$ avec $E^{\prime}$ dual à $E$. Les éléments de la classe d'équivalence de $(0, E)$ sont les couples $\left(0, E_{1}\right)$ avec $E_{1}$ dans la classe d'équivalence de $E$. Enfin les éléments de la classe d'équivalence d'un couple $\left(E^{\prime}, E^{\prime}\right)$ sont les couples $\left(E_{1}^{\prime}, E_{1}^{\prime}\right)$ avec $E_{1}^{\prime}$ dans la classe d'équivalence de $E^{\prime}$. Il reste donc à démontrer cette seconde partie.

La Proposition 2.5 montre que si $(B, E)$ admet un dual, alors $E$ est de type (D), on a $m>n$, et les duaux de $(B, E)$ sont des couples 
$\left(B^{\prime}, E^{\prime}\right)$ de $k$-sous-espaces de $K^{m-n}$, avec $B^{\prime} \subset E^{\prime}, B^{\prime}$ de dimension $m-q$, et $E^{\prime}$ de type (D), de dimension $m$. Réciproquement, supposons que $E$ soit de type (D). Cela implique $m>n$. Soient $f: K^{m} \rightarrow K^{n}$ une application linéaire telle que $f\left(k^{m}\right)=E$, et $U$ un sous-espace de $k^{m}$ tel que $f(U)=B$. Puisque $E$ est de type (D), on a $f\left(K^{m}\right)=\langle E\rangle_{K}=K^{n}$. Donc $f$ est surjective, et on peut construire une application linéaire surjective $f^{\prime}: K^{m} \rightarrow K^{m-n}$, de noyau $(\operatorname{ker} f)^{\perp}$. Soit $U^{\prime}$ le sous-espace de $k^{m}$ orthogonal à $U$. On pose $B^{\prime}=f^{\prime}\left(U^{\prime}\right)$ et $E^{\prime}=f^{\prime}\left(k^{m}\right)$. En vertu du Lemme $2.2, E^{\prime}$ est de dimension $m$. Donc les couples $(B, E)$ et $\left(B^{\prime}, E^{\prime}\right)$ sont duaux. La dernière assertion de la proposition découle du Lemme 2.6.

Proposition 2.8. - Soient $n_{1}, n_{2}$ des entiers positifs, $m$ leur somme, $\left(u_{1}, \ldots, u_{n_{1}}\right)$ une base de $K^{n_{1}},\left(v_{1}, \ldots, v_{n_{2}}\right)$ une base de $K^{n_{2}}$, et $a_{i j}$ pour $i=1, \ldots, n_{1}$ et $j=1, \ldots, n_{2}$ des éléments de $K$. On pose

$$
\begin{aligned}
& E_{1}=\left\langle u_{1}, \ldots, u_{n_{1}}, a_{11} u_{1}+\cdots+a_{n_{1} 1} u_{n_{1}}, \ldots, a_{1 n_{2}} u_{1}+\cdots+a_{n_{1} n_{2}} u_{n_{1}}\right\rangle_{k}, \\
& E_{2}=\left\langle v_{1}, \ldots, v_{n_{2}}, a_{11} v_{1}+\cdots+a_{1 n_{2}} v_{n_{2}}, \ldots, a_{n_{1} 1} v_{1}+\cdots+a_{n_{1} n_{2}} v_{n_{2}}\right\rangle_{k} .
\end{aligned}
$$

Alors $E_{1}$ est un $k$-sous-espace minimal de $K^{n_{1}}$ de dimension $m$ si et seulement si $E_{2}$ est un $k$-sous-espace étoilé de $K^{n_{2}}$ de dimension $m$. Enfin, soit $\mathcal{A}$ un sous-ensemble de $K^{n_{1} n_{2}}$. Désignons par $\mathcal{E}_{1}$ (resp. $\mathcal{E}_{2}$ ) l'ensemble que décrit $E_{1}\left(\right.$ resp. $\left.E_{2}\right)$ lorsque $\left(u_{1}, \ldots, u_{n_{1}}\right)\left(\right.$ resp. $\left.\left(v_{1}, \ldots, v_{n_{2}}\right)\right)$ parcourt l'ensemble des bases de $K^{n_{1}}\left(\right.$ resp. $\left.K^{n_{2}}\right)$ sur $K$. Alors les conditions suivantes sont équivalentes:

(i) $\mathcal{E}_{1}$ est l'ensemble des $k$-sous-espaces minimaux de $K^{n_{1}}$ de dimension $m$;

(ii) $\mathcal{E}_{2}$ est l'ensemble des $k$-sous-espaces étoilés de $K^{n_{2}}$ de dimension $m$.

Démonstration. - Soit $\Psi_{1}$ (resp. $\Psi_{2}$ ) l'automorphisme de $K^{n_{1}}$ (resp. $\left.K^{n_{2}}\right)$ qui applique la base standard de $K^{n_{1}}\left(\right.$ resp. $\left.K^{n_{2}}\right) \operatorname{sur}\left(u_{1}, \ldots, u_{n_{1}}\right)$ (resp. $\left.\left(v_{1}, \ldots, v_{n_{2}}\right)\right)$, et soit $\rho: K^{n_{2}} \rightarrow K^{n_{1}}$ l'application linéaire dont la matrice relative aux bases standards de $K^{n_{1}}$ et de $K^{n_{2}}$ est $\left(a_{i j}\right)$. On considère les applications linéaires $f_{i}: K^{n_{1}} \times K^{n_{2}} \rightarrow K^{n_{i}}(i=1,2)$ données par

$$
f_{1}(x, y)=\Psi_{1}(x+\rho(y)) \quad \text { et } \quad f_{2}(x, y)=\Psi_{2}\left(y-{ }^{t} \rho(x)\right)
$$

pour tout $x \in K^{n_{1}}$ et $y \in K^{n_{2}}$. Elles appliquent $k^{n_{1}} \times k^{n_{2}}$ sur $E_{1}$ et $E_{2}$ respectivement. De plus elles sont surjectives, et leurs noyaux sont :

$$
\operatorname{ker} f_{1}=\left\{(-\rho(y), y) ; y \in K^{n_{2}}\right\} \quad \text { et } \quad \operatorname{ker} f_{2}=\left\{\left(x,{ }^{t} \rho(x)\right) ; x \in K^{n_{1}}\right\} \text {. }
$$


Ceux-ci sont supplémentaires orthogonaux en vertu de l'égalité

$$
((x, y),(-\rho(z), z))_{m}=-(x, \rho(z))_{n_{1}}+(y, z)_{n_{2}}=\left(y-{ }^{t} \rho(x), z\right)_{n_{2}},
$$

vérifiée pour tout $(x, y) \in K^{n_{1}} \times K^{n_{2}}$, et $z \in K^{n_{2}}$. Ainsi le Lemme 2.2 s'applique. Il montre que $E_{1}$ est de type (D) et de dimension $m$ si et seulement si $E_{2}$ est de type (D) et de dimension $m$. Cette condition est nécessaire pour que $E_{1}$ soit minimal de dimension $m$, ou que $E_{2}$ soit étoilé de dimension $m$. Si elle est remplie, alors $E_{1}$ et $E_{2}$ sont duaux. Dans ce cas, la Proposition 2.5 montre que $E_{1}$ est minimal de dimension $m$ si et seulement si $E_{2}$ est étoilé de dimension $m$. Cela démontre la première partie de la proposition.

Supposons que la condition (i) soit vérifiée. La première partie de la démonstration montre alors que chaque élément de $\mathcal{E}_{2}$ est dual à un élément de $\mathcal{E}_{1}$, et vice versa. Comme $\mathcal{E}_{2}$ est une réunion de classes d'équivalence, on en déduit, grâce à la Proposition 2.7, que $\mathcal{E}_{2}$ est l'ensemble des $k$-sous-espaces de dimension $m$ de $K^{n_{2}}$ qui sont duaux à un élément de $\mathcal{E}_{1}$. Selon les Propositions 2.5 et 2.7, cela entraîne (ii). On montre de même que (ii) implique (i).

\section{Sous-espaces étoilés relatifs}

On commence par deux critères :

Lemme 3.1. - Soient $n$ un entier positif, et $B \subset E$ des $k$-sousespaces de dimension finie de $K^{n}$, avec $\langle B\rangle_{K}=K^{n}$. Alors $E$ est étoilé relativement à $B$ si et seulement si $E \cap\langle u\rangle_{K}$ est de dimension $\geq 2$ sur $k$ pour tout $u \in B$ non nul.

Démonstration. - Si la condition est remplie, on a l'inclusion $E_{\text {ass } / k} \subset\left(\langle B\rangle_{K}\right)^{\perp}=0$. Alors $E$ est de type (D). Donc la condition est suffisante. Elle est nécessaire par définition.

Lemme 3.2. - Soient $n$ un entier positif, et $E$ un $k$-sous-espace de dimension finie de $K^{n}$. Alors $E$ est étoilé si et seulement si $:\langle E\rangle_{K}=K^{n}$ et $E \cap\langle u\rangle_{K}$ est de dimension $\geq 2$ sur $k$ pour tout $u \in E$ non nul.

Démonstration. - Le Lemme 3.1 montre que la condition est suffisante. Elle est nécessaire, car si $E$ est étoilé, il est de type (D), donc il vérifie $\langle E\rangle_{K}=K^{n}$.

On montre maintenant la proposition suivante, dont découle la proposition 1.5 de [3] : 
Proposition 3.3. - Soient $n$ un entier positif, et $B \subset E$ des $k$-sousespaces de dimension finie de $K^{n}$, avec $\langle B\rangle_{K}=K^{n}$. Si $E$ est étoilé relativement à $B$, sa dimension est $\geq 2 n$.

Démonstration. - Puisque $\langle B\rangle_{K}=K^{n}$, l'espace $B$ contient une base $\left(u_{1}, \ldots, u_{n}\right)$ de $K^{n}$ sur $K$, et $E$ contient la somme directe $\left(E \cap\left\langle u_{1}\right\rangle_{K}\right)+$ $\cdots+\left(E \cap\left\langle u_{n}\right\rangle_{K}\right)$. La conclusion suit.

Pour la suite, on suppose que $k$ est un corps parfait de cardinalité infinie. Cette condition est remplie si $k$ est de caractéristique 0 , et en particulier si $k=\mathbb{Q}$. Elle implique que toute extension algébrique de $k$ est séparable ([2], ch. VII, $\S 7$, cor. de la prop. 12).

Pour chaque entier $n>0$, on munit $k^{n}$ de la topologie de Zariski, dans laquelle les fermés sont les ensembles des zéros des idéaux de $k\left[X_{1}, \ldots, X_{n}\right]$. On munit $k^{0}=\{0\}$ de la topologie discrète. Ensuite on munit chaque espace vectoriel de dimension finie sur $k$ d'une topologie de Zariski, en l'identifiant à $k^{n}$ par le choix d'une base. Cela ne dépend pas du choix de la base. Dans cette topologie, une intersection finie d'ouverts non vides est non vide. De plus, toute application $k$-linéaire entre espaces vectoriels de dimension finie sur $k$ est continue relativement à leurs topologies de Zariski. On montre :

Lemme 3.4. - Soient $U, V$ des espaces vectoriels sur $k$ de dimension finie, et $\mathcal{T}$ un sous-espace de $\operatorname{Hom}_{k}(U, V)$. L'ensemble des éléments de $\mathcal{T}$ de rang maximal constitue un ouvert de Zariski $\mathcal{O}$ de $\mathcal{T}$. Pour tout $S \in \mathcal{O}$ et tout $T \in \mathcal{T}$, on a $T(\operatorname{ker} S) \subset \operatorname{Im} S$.

Démonstration. - On peut supposer $\mathcal{T} \neq 0$. Soient $\left(T_{1}, \ldots, T_{n}\right)$ une base de $\mathcal{T}$ sur $k$, et $M_{1}, \ldots, M_{n}$ les matrices respectives de $T_{1}, \ldots, T_{n}$ relativement à un choix de bases de $U$ et $V$. Pour chaque entier $m \geq 1$, on note $I_{m}$ l'idéal de $k\left[X_{1}, \ldots, X_{n}\right]$ engendré par les mineurs d'ordre $m$ de la matrice $X_{1} M_{1}+\cdots+X_{n} M_{n}$. Soit $r$ le plus grand entier pour lequel $I_{r} \neq 0$. Les éléments de $\mathcal{T}$ sont tous de rang $\leq r$, et ceux de rang $r$ sont ceux qui s'écrivent $a_{1} T_{1}+\cdots+a_{n} T_{n}$ pour un certain $\left(a_{1}, \ldots, a_{n}\right) \in k^{n}$ qui n'est pas un zéro de $I_{r}$. Ces derniers constituent donc un ouvert non vide $\mathcal{O}$ de $\mathcal{T}$.

Soient $S \in \mathcal{O}$ et $T \in \mathcal{T}$. On choisit un supplémentaire $W$ de $\operatorname{ker} S$ dans $U$, et une base $\left(u_{1}, \ldots, u_{r}\right)$ de $W$ sur $k$. Pour tout $a \in k$ le rang de $S+a T$ est $\leq r$. Donc en désignant par $u$ un élément de $\operatorname{ker} S$, on a

$$
(S+a T)\left(u_{1}\right) \wedge \ldots \wedge(S+a T)\left(u_{r}\right) \wedge(S+a T)(u)=0
$$

TOME $118-1990-\mathrm{N}^{\circ} 2$ 
dans $\bigwedge_{k}^{r+1}(V)$. Développons le membre de gauche pour l'écrire comme combinaison linéaire de puissances de $a$ à coefficients dans $\bigwedge_{k}^{r+1}(V)$. Pour $u$ fixe, chacun de ces coefficients est nul. En considérant celui de $a$, et en tenant compte du fait que $S(u)=0$, on obtient

$$
S\left(u_{1}\right) \wedge \ldots \wedge S\left(u_{r}\right) \wedge T(u)=0
$$

donc $T(u) \in\left\langle S\left(u_{1}\right), \ldots, S\left(u_{r}\right)\right\rangle_{k}=\operatorname{Im} S$. Le choix de $u \in \operatorname{ker} S$ étant arbitraire, on a bien $T(\operatorname{ker} S) \subset \operatorname{Im} S$.

Lemme 3.5. - On conserve les notations du LEMme 3.4, et on suppose $\mathcal{T} \neq 0$. Soient $\left(T_{1}, \ldots, T_{n}\right)$ une base de $\mathcal{T}$ sur $k$, et $A_{1}, \ldots, A_{n}$ des sousensembles de $k$ de cardinalité $>\operatorname{dim}_{k} U$. Alors il existe $\left(a_{1}, \ldots, a_{n}\right) \in$ $A_{1} \times \ldots \times A_{n}$ tel que $a_{1} T_{1}+\cdots+a_{n} T_{n} \in \mathcal{O}$.

Démonstration. - Soient $r$ et $I_{r}$ comme dans la démcnstration du Lemme 3.4, pour le choix de $\left(T_{1}, \ldots, T_{n}\right)$ comme base de $\mathcal{T}$ sur $k$. Puisque $I_{r}$ est engendré par des polynômes homogènes de degré $r \leq$ $\operatorname{dim}_{k} U$, il existe un élément $\left(a_{1}, \ldots, a_{n}\right)$ de $A_{1} \times \cdots \times A_{n}$ qui n'est pas un zéro de $I_{r}$. Alors le rang de $a_{1} T_{1}+\cdots+a_{n} T_{n}$ est maximal, égal à $r$.

On utilisera la conséquence suivante du LEMme 3.4 :

Lemme 3.6. - Soient $n$ un entier positif, et $B \subset E$ des $k$-sous-espaces de dimension finie de $K^{n}$. Soient $F$ une extension de $k$ contenue dans $K$, et $\mathcal{O}$ l'ensemble des éléments non nuls $u$ de $B$ pour lesquels la dimension de $E \cap\langle u\rangle_{F}$ sur $k$ est minimale. Alors $\mathcal{O}$ est un ouvert de Zariski de $B$. Pour tout $u \in \mathcal{O}$ et tout $v \in E \cap\langle u\rangle_{F}$, on a $v \wedge_{F} B \subset u \wedge_{F} E$ dans $K^{n} \wedge_{F} K^{n}$.

Démonstration. - On peut supposer $\operatorname{dim}_{F}\langle B\rangle_{F}>1$. Soit $W$ le $k$ sous-espace de $K^{n} \wedge_{F} K^{n}$ engendré par les produits extérieurs $u \wedge_{F} v$ avec $u \in B$ et $v \in E$. On considère l'application $k$-linéaire $\theta: B \rightarrow \operatorname{Hom}_{k}(E, W)$ donnée $\operatorname{par}(\theta(u))(v)=u \wedge_{F} v$ pour tout $u \in B$ et $v \in E$. Pour $u \in B$ avec $u \neq 0$, le noyau de $\theta(u)$ est $E \cap\langle u\rangle_{F}$, contenu strictement dans $E$, le noyau de $\theta(0)$. Alors $\mathcal{O}$ est aussi l'ensemble des éléments $u$ de $B$ pour lesquels le rang de $\theta(u)$ est maximal. Suivant le Lemme 3.4 , cela entraîne que $\mathcal{O}$ est un ouvert de $B$, en tant qu'image réciproque par $\theta$ d'un ouvert de $\theta(B)$. Le même lemme montre que, pour tout $u \in \mathcal{O}$, tout $v \in E \cap\langle u\rangle_{F}$ et tout $u^{\prime} \in B$, on a $\left(\theta\left(u^{\prime}\right)\right)(v) \in \operatorname{Im}(\theta(u))$, d'où $v \wedge_{F} B \subset u \wedge_{F} E$.

Quant au Lemme 3.5, il fournit le critère suivant :

Proposition 3.7. - Soient $n$ un entier positif, et $B \subset E$ des $k$ sous-espaces de $K^{n}$ de dimension finie, avec $B \neq 0$. Supposons que $E$ 
soit de type (D) dans $K^{n}$, et désignons par $m$ et $m+q$ les dimensions respectives de $B$ et de $E$. Soient $\left(u_{1}, \ldots, u_{m}\right)$ une base de $B$ sur $k$, et $A_{1}, \ldots, A_{m}$ des sous-ensembles de $k$ de cardinalité $>m+q$. Alors $E$ est un $k$-sous-espace étoilé de $K^{n}$ relativement $\grave{a} B$ si et seulement si la dimension de $E \cap\left\langle a_{1} u_{1}+\cdots+a_{m} u_{m}\right\rangle_{K}$ sur $k$ est $\geq 2$, pour tout $\left(a_{1}, \ldots, a_{m}\right) \in A_{1} \times \cdots \times A_{m}$ non $n u l$.

Démonstration. - La condition est nécessaire. Supposons qu'elle soit remplie. Si $\operatorname{dim}_{K}\langle B\rangle_{K}=1$, on obtient sans peine que $E$ est étoilé relativement à $B$. Cela permet de supposer $\operatorname{dim}_{K}\langle B\rangle_{K}>1$. Dans ce cas, soient $\mathcal{O}, W$ et $\theta$ comme dans le Lemme 3.6 et sa démonstration, pour le choix de $F=K$. Le Lemme 3.5 montre que $\mathcal{O}$ contient un élément $u_{0}$ de la forme $a_{1} u_{1}+\cdots+a_{m} u_{m}$ pour un certain $\left(a_{1}, \ldots, a_{m}\right) \in A_{1} \times \cdots \times A_{m}$. Puisque $0 \notin \mathcal{O}$, on a $\left(a_{1}, \ldots, a_{m}\right) \neq 0$. Alors, par hypothèse, la dimension de $E \cap\left\langle u_{0}\right\rangle_{K}$ sur $k$ est $\geq 2$. Comme $\mathcal{O}$ consiste des éléments non nuls $u$ de $B$ pour lesquels la dimension de $E \cap\langle u\rangle_{K}$ sur $k$ est minimale, cela implique que $E$ est étoilé relativement à $B$. La condition est donc suffisante.

On conclut ce paragraphe par deux théorèmes. Ils admettent respectivement pour corollaire les théorèmes 1.6 et 1.7 de [3].

ThÉORÈmE 3.8. - Soient $n$ un entier $\geq 2$, et $E$ un $k$-sous-espace de $K^{n}$ de dimension finie. Alors $E$ est étoilé si et seulement si $:\langle E\rangle_{K}=K^{n}$ et $i l$ existe une extension $F$ de $k$, de degré fini $d \geq 2$, contenue dans $K$, vérifiant

$$
\operatorname{dim}_{k}\langle E\rangle_{F} \leq \operatorname{dim}_{k}(E)+d-2 .
$$

Démonstration. - Supposons d'abord $\langle E\rangle_{K}=K^{n}$, et l'existence d'une extension $F$ de $k$, de degré fini $d \geq 2$, contenue dans $K$, vérifiant (1). Pour tout élément non nul $u$ de $E$, on a $E+\langle u\rangle_{F} \subset\langle E\rangle_{F}$, donc $\operatorname{dim}_{k}\left(E \cap\langle u\rangle_{F}\right) \geq 2$. En vertu du Lemme 3.2, cela implique que $E$ est étoilé.

Réciproquement, supposons que $E$ soit un $k$-sous-espace étoilé de $K^{n}$. Pour chaque extension $F$ de $k$ contenue dans $K$, on désigne par $\mathcal{O}_{F}$ l'ensemble des éléments non nuls $u$ de $E$ pour lesquels la dimension de $E \cap\langle u\rangle_{F}$ sur $k$ est minimale, et $e_{F}$ cette dimension minimale. On pose aussi $M(u)=\{t \in K ; t u \in E\}$ pour chaque $u \in E$. Alors, pour une extension $F$ donnée, $\mathcal{O}_{F}$ consiste des éléments $u$ de $E$ pour lesquels $\operatorname{dim}_{k}(F \cap M(u))=e_{F}$. D'après le Lemme 3.2, le fait que $E$ soit étoilé se traduit $\operatorname{par}\langle E\rangle_{K}=K^{n}$ et $e_{K} \geq 2$.

Le LeMme 3.6 montre que, pour $F$ donnée, l'ensemble $\mathcal{O}_{F}$ est un ouvert de $E$, et que, pour $u \in \mathcal{O}_{F}$, le $k$-sous-espace $u \wedge_{F} E$ de $K^{n} \wedge_{F} K^{n}$ est

$$
\text { TOME } 118-1990-\mathrm{N}^{\circ} 2
$$


fermé sous la multiplication par les éléments de $F \cap M(u)$. Alors, on peut considérer $u \wedge_{F} E$ comme sous-espace vectoriel de $K^{n} \wedge_{F} K^{n}$ sur l'extension de $k$ engendrée par $F \cap M(u)$. Or $u \wedge_{F} E$ est de dimension finie sur $k$, non nulle puisque $\langle E\rangle_{K}=K^{n}$ et $n \geq 2$. Donc l'extension en question est algébrique sur $k$, de degré fini.

Soient $L$ la fermeture algébrique de $k$ dans $K$, et $F^{\prime}$ une extension de $k$ contenue dans $L$, de degré fini sur $k$, telle que $\operatorname{dim}_{L}\langle E\rangle_{L}=\operatorname{dim}_{F^{\prime}}\langle E\rangle_{F^{\prime}}$. On choisit $u \in \mathcal{O}_{K} \cap \mathcal{O}_{F^{\prime}}$. Comme $u \in \mathcal{O}_{K}$, les considérations précédentes donnent $M(u) \subset L$. En vertu du choix de $F^{\prime}$, cela implique $M(u) \subset F^{\prime}$. On en déduit $e_{F^{\prime}}=e_{K}$.

Soit $F$ une extension de $k$, contenue dans $F^{\prime}$, de degré minimal sur $k$, telle que $e_{F}=e_{K}$. Puisque le corps $k$ est parfait, le corps $F^{\prime}$ est une extension séparable de $k$. Il contient donc un nombre fini d'extensions de $k$. On choisit un élément $u$ dans l'intersection des ouverts $\mathcal{O}$ associés à celles-ci. Soit $F^{\prime \prime}$ l'extension de $k$ engendrée par $F \cap M(u)$. Puisque $F^{\prime \prime} \cap M(u)=F \cap M(u)$, le choix de $u$ implique $e_{F^{\prime \prime}}=e_{F}$, donc $F^{\prime \prime}=F$. Ainsi $u \wedge_{F} E$ est un $F$-sous-espace de $K^{n} \wedge_{F} K^{n}$. Cela signifie $u \wedge_{F} E=u \wedge_{F}\langle E\rangle_{F}$. En comparant les dimensions sur $k$ des deux membres de cette égalité, on obtient $\operatorname{dim}_{k} E-e_{F}=\operatorname{dim}_{k}\langle E\rangle_{F}-\operatorname{dim}_{k} F$. Puisque $e_{F}=e_{K} \geq 2$, le corps $F$ possède les propriétés annoncées.

THÉORÈME 3.9. - Soient $n$ un entier $\geq 2$, et $B \subset E$ des $k$-sous-espaces de $K^{n}$ de dimension finie. Supposons $B$ de codimension 1 dans $E$, et $\langle B\rangle_{K}=K^{n}$. Alors $E$ est étoilé relativement à $B$ si et seulement si $B$ ou E est étoilé.

Démonstration. - Il est clair que la condition est suffisante. Pour montrer qu'elle est nécessaire, supposons que $E$ soit étoilé relativement à $B$, mais que $B$ ne soit pas étoilé. Il s'agit de montrer que $E$ est étoilé.

Puisque $B$ n'est pas étoilé, l'ensemble des éléments non nuls $u$ de $B$ qui vérifient $B \cap\langle u\rangle_{K}=\langle u\rangle_{k}$ constituent un ouvert non vide $\mathcal{O}$ de $B$. Cela découle du Lemme 3.6. Pour chaque sous-corps $F$ de $K$, on note $\mathcal{O}_{F}$ l'ensemble des éléments non nuls $u$ de $B$ pour lesquels la dimension de $E \cap\langle u\rangle_{F}$ sur $k$ est minimale, et $e_{F}$ cette dimension minimale. Le même lemme montre que $\mathcal{O}_{F}$ est un ouvert de $B$.

Soit $F$ une extension de $k$ contenue dans $K$, avec $e_{F} \geq 2$. On choisit $u \in \mathcal{O} \cap \mathcal{O}_{F}$. Alors $B+\left(E \cap\langle u\rangle_{F}\right)$ coïncide avec $E$ puisqu'il contient $B$ proprement. On en déduit $u \wedge_{F} B=u \wedge_{F} E$, et le Lemme 3.6 montre que le $k$-sous-espace $u \wedge_{F} E$ de $K^{n} \wedge_{F} K^{n}$ est fermé sous la multiplication par les éléments $t$ de $F$ pour lesquels $t u \in E$. Ainsi, on peut raisonner comme dans la démonstration du THÉORÈme 3.8, en prenant soin chaque fois de 
choisir $u \in \mathcal{O}$. On en déduit l'existence d'une extension algébrique $F$ de $k$, de degré fini $d \geq 2$, contenue dans $K$, vérifiant $\operatorname{dim}_{k} E \geq \operatorname{dim}_{k}\langle E\rangle_{F}-d+2$. Suivant le ThÉORÈme 3.8 , cela signifie que $E$ est étoilé.

\section{Sous-espaces minimaux relatifs}

On démontre d'abord l'énoncé dual de la Proposition 3.3. La proposition 1.8 de [3] s'en déduit.

Proposition 4.1. - Soient $n$ un entier positif, $E$ un $k$-sous-espace de $K^{n}$ de dimension finie, et $B$ un sous-espace de $E$ engendré par des éléments linéairement indépendants sur $K$. Si E est minimal relativement $\grave{a} B$, sa dimension est $\leq 2 n$.

Démonstration. - Supposons que $E$ soit minimal relativement à $B$, et soit $m$ sa dimension sur $k$. Suivant les Propositions 2.5 et 2.7 , on a $m>n$, et le couple $(B, E)$ admet pour dual un couple $\left(B^{\prime}, E^{\prime}\right)$ de $k$-sous-espaces de $K^{m-n}$, avec $B^{\prime} \subset E^{\prime},\left\langle B^{\prime}\right\rangle_{K}=K^{m-n}$, et $E^{\prime}$ étoilé relativement à $B^{\prime}$, de dimension $m$. Alors la Proposition 3.3 appliquée au couple $\left(B^{\prime}, E^{\prime}\right)$ livre $m \geq 2(m-n)$, d'où $m \leq 2 n$.

La dimension sur $k$ d'un $k$-sous-espace minimal de $K^{n}$ est donc $\leq 2 n$. Elle est aussi $\geq n+1$, car un tel $k$-sous-espace de $K^{n}$ est d'abord de type (D).

Pour la suite, on suppose comme au $\S 3$, que $k$ est un corps parfait de cardinalité infinie. La proposition suivante indique une circonstance où la dimension d'un $k$-sous-espace minimal de $K^{n}$ est $n+1$ (la proposition 1.10 de [3] s'en déduit) :

Proposition 4.2. - Soient $n$ un entier positif, et $F$ une extension de $k$ contenue dans $K$. Supposons que $k$ soit algébriquement clos dans $F$. Alors, s'il existe un $k$-sous-espace minimal de $K^{n}$ contenu dans $F^{n}$, sa dimension est $n+1$.

Démonstration. - Supposons qu'il existe un $k$-sous-espace minimal $E$ de $K^{n}$ contenu dans $F^{n}$, et soit $m$ sa dimension. Alors $E$ est aussi un $k$ sous-espace minimal de $F^{n}$. En tant que tel, suivant les Propositions 2.5 et 2.7 , il vérifie $m \geq n+1$, et admet pour dual un $k$-sous-espace étoilé de $F^{m-n}$ de dimension $m$. En vertu du ThÉorème 3.8, cela implique $m-n=1$.

Il est facile de décrire les $k$-sous-espaces minimaux de $K^{n}$ de dimension $n+1$ (comparer à [1], $\S 1, \mathrm{n}^{\circ} 3$, prop. 7 , cor. 2$)$ :

TOME $118-1990-\mathrm{N}^{\circ} 2$ 
Proposition 4.3. - Soit $n$ un entier positif. Les $k$-sous-espaces minimaux de $K^{n}$ de dimension $n+1$, s'il en existe, sont les $k$-sous-espaces de $K^{n}$ qui s'écrivent $\left\langle u_{1}, \ldots, u_{n}, t_{1} u_{1}+\cdots+t_{n} u_{n}\right\rangle_{k}$ pour une base $\left(u_{1}, \ldots, u_{n}\right)$ de $K^{n}$ sur $K$, et des éléments $t_{1}, \ldots, t_{n}$ de $K$ tels que $1, t_{1}, \ldots, t_{n}$ soient linéairement indépendants sur $k$.

Démonstration. - Cela découle immédiatement de la Proposition 2.8 appliquée au fait que les $k$-sous-espaces étoilés de $K$ de dimension $n+1$ sont les $k$-sous-espaces de $K$ qui s'écrivent $\left\langle v, t_{1} v, \ldots, t_{n} v\right\rangle_{k}$ pour une base $(v)$ de $K$, et des éléments $t_{1}, \ldots, t_{n}$ de $K$ tels que $1, t_{1}, \ldots, t_{n}$ soient linéairement indépendants sur $k$.

Le lemme suivant précise l'énoncé du ThÉorème 3.8 en vue des applications qui vont suivre :

LEMmE 4.4. - Soient $n$ un entier $\geq 2$, et $E$ un $k$-sous-espace de $K^{n}$ de dimension finie $m$ sur $k$. Alors $E$ est étoilé si et seulement si $E \subset$ $\left\langle u_{1}, \ldots, u_{l}\right\rangle_{F}$ pour une extension $F$ de $k$, contenue dans $K$, de degré finid sur $k$ vérifiant $2 \leq d \leq(m-2) /(n-1)$ et ne divisant pas $m-1$, et une famille d'éléments $u_{1}, \ldots, u_{l}$ de $K^{n}$, linéairement indépendants sur $F$, contenant une base de $K^{n}$ sur $K$, de cardinalité l égale à la partie entière $d e(m+d-2) / d$.

Démonstration. - Supposons d'abord que $E$ soit étoilé. Alors il contient une base $\left(u_{1}, \ldots, u_{n}\right)$ de $K^{n}$ sur $K$, et d'après le ThÉORÈmE 3.8 , il existe une extension $F$ de $k$, contenue dans $K$, de degré fini $d \geq 2$ sur $k$, vérifiant

$$
\operatorname{dim}_{k}\langle E\rangle_{F} \leq m+d-2
$$

Comme $u_{1}, \ldots, u_{n}$ sont linéairement indépendants sur $K$, on peut les compléter en une base $\left(u_{1}, \ldots, u_{l}\right)$ de $\langle E\rangle_{F}$ sur $F$. Alors l'inégalité (1) se réécrit $d l \leq m+d-2$. Comme $l \geq n$, cela implique $d \leq(m-2) /(n-1)$. Comme $\langle E\rangle_{F}$ contient $E$, on a aussi $m \leq d l$. Donc $l$ est la partie entière de $(m+d-2) / d$, et $d$ ne divise pas $m-1$. Ainsi les conditions du lemme sont nécessaires.

Supposons maintenant que ces conditions soient remplies. On a $\langle E\rangle_{F} \subset$ $\left\langle u_{1}, \ldots, u_{l}\right\rangle_{F}$. Comme la dimension sur $k$ du membre de gauche de cette inclusion est $\geq m$, que celle du membre de droite est $\leq m+d-2$, et que toutes deux sont multiples de $d$, ces dimensions coïncident. Donc (1) est vérifiée, et on a $\langle E\rangle_{F}=\left\langle u_{1}, \ldots, u_{l}\right\rangle_{F}$. Cette dernière égalité implique $\langle E\rangle_{K}=K^{n}$ puisque la famille $u_{1}, \ldots, u_{l}$ contient une base de $K^{n}$ sur $K$. Alors $E$ est étoilé en vertu du ThÉORÈme 3.8 . 
Proposition 4.5. - Soient $n$ un entier $\geq 2$, et $E$ un $k$-sous-espace de $K^{n}$. Les conditions suivantes sont équivalentes :

(i) $E$ est étoilé de dimension $2 n$;

(ii) $E$ est minimal de dimension $2 n$;

(iii) E est étoilé et minimal;

(iv) $E=\left\langle u_{1}, \ldots, u_{n}\right\rangle_{F}$ pour une base $\left(u_{1}, \ldots, u_{n}\right)$ de $K^{n}$ sur $K$, et une extension quadratique $F$ de $k$ contenue dans $K$.

Démonstration. - L'équivalence entre les conditions (i) et (iv) est une conséquence directe du LEMme 4.4. Ainsi les $k$-sous-espaces étoilés de $K^{n}$ de dimension $2 n$ sont les $k$-sous-espaces de $K^{n}$ qui s'écrivent $\left\langle u_{1}, \ldots, u_{n}, t u_{1}, \ldots, t u_{n}\right\rangle_{k}$ pour une base $\left(u_{1}, \ldots, u_{n}\right)$ de $K^{n}$, et un élément $t$ de $K$, algébrique de degré 2 sur $k$. En appliquant la Proposition 2.8, on trouve que les $k$-sous-espaces minimaux de $K^{n}$ de dimension $2 n$ ont la même forme. Donc les conditions (i) et (ii) sont équivalentes. Puisqu'elles entraînent la condition (iii), il reste seulement à démontrer que, si $E$ est étoilé et minimal, sa dimension est $2 n$. Cela découle des Propositions 3.3 et 4.1 .

Lemme 4.6. - Pour $n \geq 3$, il n'existe pas de k-sous-espace étoilé de $K^{n}$ de dimension $2 n+1$. Les $k$-sous-espaces étoilés de $K^{2}$ de dimension 5, s'il en existe, sont les $k$-sous-espaces de $K^{2}$ qui s'écrivent $\left\langle u_{1}, u_{2}, t u_{1}, t u_{2}, t^{2} u_{2}\right\rangle_{k}$, pour une base $\left(u_{1}, u_{2}\right)$ de $K^{2}$ sur $K$, et un élément $t$ de $K$ algébrique de degré 3 sur $k$.

Démonstration. - Soient $n$ un entier $\geq 2$, et $E$ un $k$-sous-espace de $K^{n}$ de dimension $2 n+1$. Le Lemme 4.4 montre que $E$ est étoilé si et seulement si $n=2$ et il existe une extension cubique $F$ de $k$, contenue dans $K$, et une base $\left(u_{1}, u_{2}\right)$ de $K^{2}$ sur $K$, telle que $E$ soit de codimension 1 dans $\left\langle u_{1}, u_{2}\right\rangle_{F}$. Si tel est le cas, $E$ est donné par

$$
E=\left\{a_{1} u_{1}+a_{2} u_{2} ; a_{1}, a_{2} \in F \text { et } \operatorname{Tr}_{F / k}\left(a_{1} b_{1}+a_{2} b_{2}\right)=0\right\}
$$

pour certains $b_{1}, b_{2} \in F$ non tous nuls, où $\operatorname{Tr}_{F / k}$ désigne la trace de $F$ sur $k$. En effet, $F$ est une extension séparable de $k$ puisque le corps $k$ est parfait. On pose $u^{\prime}=b_{2} u_{1}-b_{1} u_{2}$. Alors $\left\langle u^{\prime}\right\rangle_{F}$ est contenu dans $E$, et $E$ s'écrit $E=\langle u, t u\rangle_{k} \oplus\left\langle u^{\prime}\right\rangle_{F}$ pour un certain $u \in E$, et un certain $t \in F \backslash k$. Puisque $\langle E\rangle_{K}=K^{2}$, les vecteurs $u, u^{\prime}$ constituent une base de $K^{2}$. Enfin, puisque $t \notin k$, on a $F=k(t)$, et $E$ s'écrit aussi $E=\left\langle u, t u, u^{\prime}, t u^{\prime}, t^{2} u^{\prime}\right\rangle_{k}$. Réciproquement, si $n=2$ et si $E$ s'écrit $\left\langle u_{1}, u_{2}, t u_{1}, t u_{2}, t^{2} u_{2}\right\rangle_{k}$, pour une base $\left(u_{1}, u_{2}\right)$ de $K^{2}$ sur $K$, et un élément $t$ de $K$ algébrique de degré 3

$$
\text { TOME } 118-1990-\mathrm{N}^{\circ} 2
$$


sur $k$, alors $E$ est de codimension 1 dans $\left\langle u_{1}, u_{2}\right\rangle_{F}$, où $F$ est le corps $k(t)$, de degré 3 sur $k$. Alors, suivant le Lemme 4.4, $E$ est un $k$-sous-espace étoilé de $K^{2}$.

Proposition 4.7. - Pour $n \geq 4$, il n'existe pas de $k$-sous-espace minimal de $K^{n}$ de dimension $2 n-1$. Les $k$-sous-espaces minimaux de $K^{3}$ de dimension 5 , s'il en existe, sont les $k$-sous-espaces de $K^{3}$ qui s'écrivent $\left\langle v_{1}, v_{2}, v_{3}, t v_{1}, t v_{2}+t^{2} v_{3}\right\rangle_{k}$, pour une base $\left(v_{1}, v_{2}, v_{3}\right)$ de $K^{3}$ sur $K$, et un élément $t$ de $K$ algébrique de degré 3 sur $k$.

Démonstration. - En vertu des Propositions 2.5 et 2.7, un $k$-sousespace de $K^{n}$ de dimension $2 n-1$ est minimal si et seulement s'il admet pour dual un $k$-sous-espace étoilé de $K^{n-1}$ de dimension $2 n-1$. Le Lemme 4.6 montre que, pour $n \geq 4$, l'existence de ce dernier est exclue, alors l'existence du premier l'est aussi. Suivant la Proposition 2.8, les dernières assertions de la proposition et du Lemme 4.6 sont équivalentes.

Des Propositions 4.5 et 4.7, on retient la conséquence suivante, dont découle la proposition 1.9 de [3] :

Corollaire 4.8. - Soit $n$ un entier positif. Supposons qu'il existe un $k$-sous-espace minimal $E$ de $K^{n}$, et soit $m$ sa dimension. Si $n \geq 2$ et $m=2 n$, ou si $n \geq 3$ et $m=2 n-1$, alors il existe deux éléments de $E$ qui sont linéairement indépendants sur $k$, mais linéairement dépendants sur la clôture algébrique de $k$ dans $K$.

L'énoncé suivant est dual du ThÉORÈmE 3.9, et le théorème 1.11 de [3] s'en déduit :

ThÉORÈme 4.9. - Soient $n$ un entier $\geq 2$, et $B \subset E$ des $k$-sousespaces de dimension finie de $K^{n}$. Supposons $B$ de dimension 1 , et $E$ de dimension $m \geq n+2$. Soit $\pi: K^{n} \rightarrow K^{n-1}$ une application $K$-linéaire de noyau $\langle B\rangle_{K}$. Alors $E$ est un $k$-sous-espace minimal de $K^{n}$ relativement $\grave{a} B$ si et seulement si, ou bien $E$ est un $k$-sous-espace minimal de $K^{n}$, ou bien $E$ est un $k$-sous-espace de type (D) de $K^{n}$, et $\pi(E)$ est un $k$-sousespace minimal de dimension $m-1$ de $K^{n-1}$.

Démonstration. - Si $E$ est minimal, il est minimal relativement à $B$. Donc pour montrer que la condition est suffisante, on peut supposer que $E$ est de type (D), et que $\pi(E)$ est un $k$-sous-espace minimal de dimension $m-1$ de $K^{n-1}$. Alors $B$ est le noyau de la restriction de $\pi$ à $E$. Soit $E_{1}$ un sous-espace de $E$ de dimension $m-1$ contenant $B$. Son image par $\pi$ est un sous-espace propre de $\pi(E)$. Donc elle n'est pas de type (D) dans $K^{n-1}$. Alors $E_{1}$ n'est pas de type (D) dans $K^{n}$, $\operatorname{car}^{t} \pi$ est injective et applique 
$\left(\pi\left(E_{1}\right)\right)_{\text {ass } / k}$ dans $\left(E_{1}\right)_{\text {ass } / k}$. Le choix de $E_{1}$ étant arbitraire, $E$ est un $k$-sous-espace minimal de $K^{n}$ relativement à $B$.

Pour montrer la réciproque, supposons que $E$ soit un $k$-sous-espace minimal de $K^{n}$ relativement à $B$, mais qu'il ne soit pas minimal. Puisque $E$ est de type (D), il s'agit de montrer que $\pi(E)$ est un $k$-sous-espace minimal de dimension $m-1$ de $K^{n-1}$. Soit $f: K \times K^{m-1} \rightarrow K^{n}$ une application linéaire surjective telle que $f(k \times 0)=B$ et $f\left(k \times k^{m-1}\right)=E$, et soit $f^{\prime}: K \times K^{m-1} \rightarrow K^{m-n}$ une application linéaire surjective de noyau $(\operatorname{ker} f)^{\perp}$. On pose $B^{\prime}=f^{\prime}\left(0 \times k^{m-1}\right)$ et $E^{\prime}=f^{\prime}\left(k \times k^{m-1}\right)$. Puisque $E$ est un $k$-sous-espace de type (D) de $K^{n}$ de dimension $m$, le Lemme 2.2 montre que $E^{\prime}$ est un $k$-sous-espace de type (D) de $K^{m-n}$ de dimension $m$. Donc les couples $(B, E)$ et $\left(B^{\prime}, E^{\prime}\right)$ sont duaux. En vertu de la Proposition 2.5, cela implique que $E^{\prime}$ est étoilé relativement à $B^{\prime}$, mais non étoilé, et que $B^{\prime}$ est un sous-espace de codimension 1 de $E^{\prime}$, vérifiant $\left\langle B^{\prime}\right\rangle_{K}=K^{m-n}$. Donc, suivant le ThÉorème $3.9, B^{\prime}$ est un $k$-sous-espace étoilé de $K^{m-n}$. Soit $\Phi: K^{m-1} \rightarrow K \times K^{m-1}$ l'application linéaire donnée par $\Phi(x)=(0, x)$ pour tout $x \in K^{m-1}$. On pose $g=\pi \circ f \circ \Phi: K^{m-1} \rightarrow K^{n-1}$ et $g^{\prime}=f^{\prime} \circ \Phi: K^{m-1} \rightarrow K^{m-n}$. Ce sont deux applications linéaires de noyaux supplémentaires orthogonaux dans $K^{m-1}$. En effet, leurs noyaux sont respectivement les images réciproques par $\Phi$ de $\operatorname{ker}(\pi \circ f)=(\operatorname{ker} f)+(K \times 0)$ et $\left(\operatorname{ker} f^{\prime}\right) \cap\left(0 \times K^{m-1}\right)$, et ceuxci sont supplémentaires orthogonaux dans $K^{m}$. Puisque $g^{\prime}\left(k^{m-1}\right)=B^{\prime}$ est un $k$-sous-espace étoilé de dimension $m-1$ de $K^{m-n}$, le LEMmE 2.2 montre que $g\left(k^{m-1}\right)=\pi(E)$ est un $k$-sous-espace de type (D) et de dimension $m-1$ de $K^{n-1}$. Donc $B^{\prime}$ et $\pi(E)$ sont duaux. Puisque $B^{\prime}$ est étoilé, la Proposition 2.5 montre que $\pi(E)$ est minimal.

On conclut par le critère suivant, dont découle la proposition 1.12 de $[3]$ :

Proposition 4.10. - Soient $n$ un entier positif, et $B \subset E$ des $k$ sous-espaces de $K^{n}$ de dimension finie, avec $B \neq E$. Supposons que $E$ soit de type (D) dans $K^{n}$, et désignons par $q$ et $m+q$ les dimensions respectives de $B$ et de $E$. Soit $\left(\phi_{1}, \ldots, \phi_{m}\right)$ une base du sous-espace de $\operatorname{Hom}_{k}(E, k)$ constitué des applications linéaires qui s'annulent sur $B$, et soient $A_{1}, \ldots, A_{m}$ des sous-ensembles de $k$ de cardinalité $>m+q$. Alors $E$ est un $k$-sous-espace minimal de $K^{n}$ relativement $\grave{a} B$ si et seulement si, pour tout $\left(a_{1}, \ldots, a_{m}\right) \in A_{1} \times \cdots \times A_{m}$ non nul, le noyau de $a_{1} \phi_{1}+\cdots+a_{m} \phi_{m} n^{\prime}$ est pas un $k$-sous-espace de type (D) de $K^{n}$.

Démonstration. - Soit $f: K^{m} \times K^{q} \rightarrow K^{n}$ une application linéaire telle que $f\left(0 \times k^{q}\right)=B$ et $f\left(k^{m} \times k^{q}\right)=E$, et soit $f^{\prime}: K^{m} \times K^{q} \rightarrow K^{m+q-n}$ 
une application linéaire de noyau $(\operatorname{ker} f)^{\perp}$. On pose $B^{\prime}=f^{\prime}\left(k^{m} \times 0\right)$ et $E^{\prime}=f^{\prime}\left(k^{m} \times k^{q}\right)$. En vertu du Lemme 2.2, $E^{\prime}$ est de dimension $m+q$ sur $k$. Alors les couples $(B, E)$ et $\left(B^{\prime}, E^{\prime}\right)$ sont duaux. Suivant la Proposition 2.5, cela entraîne que $E$ est un $k$-sous-espace minimal de $K^{n}$ relativement à $B$ si et seulement si $E^{\prime}$ est un $k$-sous-espace étoilé de $K^{m+q-n}$ relativement à $B^{\prime}$. Pour $i=1, \ldots, m$, on désigne par $x_{i}$ l'élément de $k^{m} \times 0$ qui vérifie $\left(x_{i}, y\right)_{m+q}=\phi_{i}(f(y))$ pour tout $y \in k^{m} \times k^{q}$, et on pose $u_{i}=f^{\prime}\left(x_{i}\right)$. Alors $\left(u_{1}, \ldots, u_{m}\right)$ constitue une base de $B^{\prime}$ sur $k$. De plus, pour $\left(a_{1}, \ldots, a_{m}\right) \in k^{m}$ non nul, le noyau de $a_{1} \phi_{1}+\cdots+a_{m} \phi_{m}$ est $f\left(\left(a_{1} x_{1}+\cdots+a_{m} x_{m}\right)^{\perp}\right)$, où $\left(a_{1} x_{1}+\cdots+a_{m} x_{m}\right)^{\perp}$ désigne le sous-espace de $k^{m} \times k^{q}$ orthogonal à $a_{1} x_{1}+\cdots+a_{m} x_{m}$. D'après le Lemme 2.3, ce noyau n'est pas un $k$-sous-espace de type (D) de $K^{n}$ si et seulement si la dimension de $E^{\prime} \cap\left\langle a_{1} u_{1}+\cdots+a_{m} u_{m}\right\rangle_{K}$ sur $k$ est $\geq 2$. La Proposition 3.7 montre que $E^{\prime}$ est étoilé relativement à $B^{\prime}$ si et seulement si cette dernière condition est remplie pour tout $\left(a_{1}, \ldots, a_{m}\right) \in A_{1} \times \cdots \times A_{m}$ non nul. La conclusion s'ensuit.

\section{BIBLIOGRAPHIE}

[1] N. Bourbaki. - Topologie générale, chap. VII. - Hermann, Paris, 1974.

[2] S. LANG. - Algebra. - Addison Wesley, Don Mills, Ontario, 1971.

[3] D. Roy. - Sous-groupes minimaux des groupes de Lie commutatifs réels et applications arithmétiques, Acta arith., t. 56, 1990, (à paraître). 